\title{
An ultrastructural study of ejaculated spermatozoa from three patients presenting total sperm immotility
}

\author{
Sousa, M. , Gouveia, M., ${ }^{* * *}$, Ferraz, L. ${ }^{* * *}$, Barros, A. ${ }^{* * * *}$, Oliveira, E. , Alves, A. , Sá, R.
}

Lab of Cell Biology, UMIB-FCT, Institute of Biomedical Sciences Abel Salazar (ICBAS), University of Porto, Rua Jorge Viterbo Ferreira 228, Porto, PORTUGAL

*** Faculty of Sciences, University of Porto, Porto, PORTUGAL

\author{
Department of Urology, Hospital Centre of Vila Nova de Gaia/Espinho, EPE, V. N. Gaia, PORTUGAL \\ ***** Centre for Reproductive Genetics Alberto Barros, Porto, PORTUGAL \\ Email: rmsa@icbas.up.pt
}

Sperm motility is fundamental for migration through the female genital tract as well as to penetrate the zona pellucida of the oocyte. In cases of absolute immotility, the ultrastructural study of spermatozoa is the only tool available to provide a diagnosis and thereafter to search for the presence of specific genetic mutations and give a prognosis to the patient and family [1].

We studied by transmission electron microscopy (TEM) semen samples from three patients with total sperm immotility in order to obtain a diagnosis for this problem.

Under informed written consent, semen was collected, allowed to liquefy, washed with SPM (Sperm preparation medium, Medicult Origio, Jyllinge, Denmark), mixed with sodium cacodylate buffer, $0.1 \mathrm{M}, \mathrm{pH}$ 7.2 (1:1) (Merck; Darmstadt, Germany) and centrifuged for $10 \mathrm{~min}$ at $1500 \mathrm{rpm}$. The pellet was then fixed with karnovsky for $2 \mathrm{~h}$, washed, post-fixed with $2 \%$ osmium tetroxide (Merck) in buffer for $2 \mathrm{~h}$ at $4^{\circ} \mathrm{C}$, washed, serially dehydrated in ethanol, equilibrated in propylene oxide (Merck), serially impregnated with propylene oxide and Epon (Sigma-Aldrich, Steinheim, Switxerland) and embedded in Epon. Semithin and ultrathin sections were prepared with a diamond knife (Diatome, Hatfield, Switzerland) on a LKB ultramicrotome (Leica Microssystems, Weltzlar, Germany). Ultrathin sections were collected on 300 mesh copper grids (Taab, Berks, England), contrasted with aqueous uranyl acetate (BDH, Poole, England) and Reynolds lead citrate (Merck), and observed in a transmission electron microscope (JEOL 100CXII, Tokyo, Japan) operated at $60 \mathrm{kV}$.

At the ultrastructural level, Patient 1 presented multiple defects attaining the midpiece, the fibers and the microtubules of the axoneme (Fig. 1A-D). In Patient 2 it was observed disruption of the axonemal microtubules (Fig. 2A-C). Patient 3 showed disorganization of the midpiece and fibers, with an intact axoneme except for the absence of dynein arms (Fig.3 A-E).

In Patient 1 no definitive diagnosis could be given as the anomalies found were multiple. For Patient 2 as the main feature was disorganization of the axoneme it may be related with mutations in CCDC (Coiled-coil domain) genes [2]. For Patient 3, the absence of dynein arms indicates mutations in DNAH (Dynein heavy chain) genes [3].

These patients may get fathering by the use of intracytoplasmic sperm injection, as selection of a near normal head will indicate correct centriole positioning as observed in all cases. To get further insight into the genetic causes, patients should be screened for mutations of the above cited genes as well as for the outer dense fibers (ODF genes) [4] and fibrous sheath (AKAP genes) [3] genes.

[1] Cabral, M. et al., Revista Internacional de Andrologia, 10, 156-159,2012.

[2] Inaba, K. Molecular Human Reproduction, 17, 524-538, 2011.

[3] Chemes, H.E., Rawe, V.Y., Human Reproduction Update, 9, 405-428, 2003.

[4] Horowitz, E. et al., Molecular Human Reproduction, 11, 307-317, 2005. 


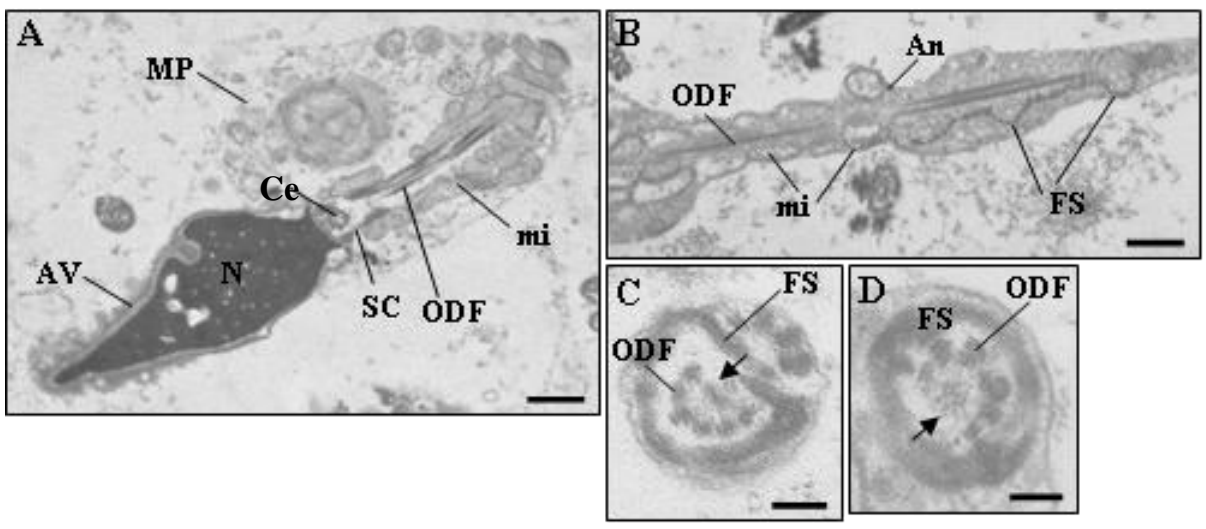

Figure 1. Patient 1 A. Normal nucleus (N), acrosomal vesicle (AV) and centriole region (Ce). Abnormal midpiece (MP) with disorganization of the mitochondrial sheath (mi) at the apical region. Striated columns (SC); outer dense fibers (ODF); B. Conserved mitochondrial sheath at distal MP with presence of the annulus (An). C. Disorganization of the fibrous sheath (FS). C, D. Abnormal number of the axonemal microtubules (arrows) and ODF. C. Missing of 3 pairs of microtubules and of 3 ODF. D. Missing of 5 ODF and 3 pairs of microtubules. Bars: $1 \mu \mathrm{m}(\mathrm{A}, \mathrm{B}) ; 0.1 \mu \mathrm{m}(\mathrm{C}, \mathrm{D})$.

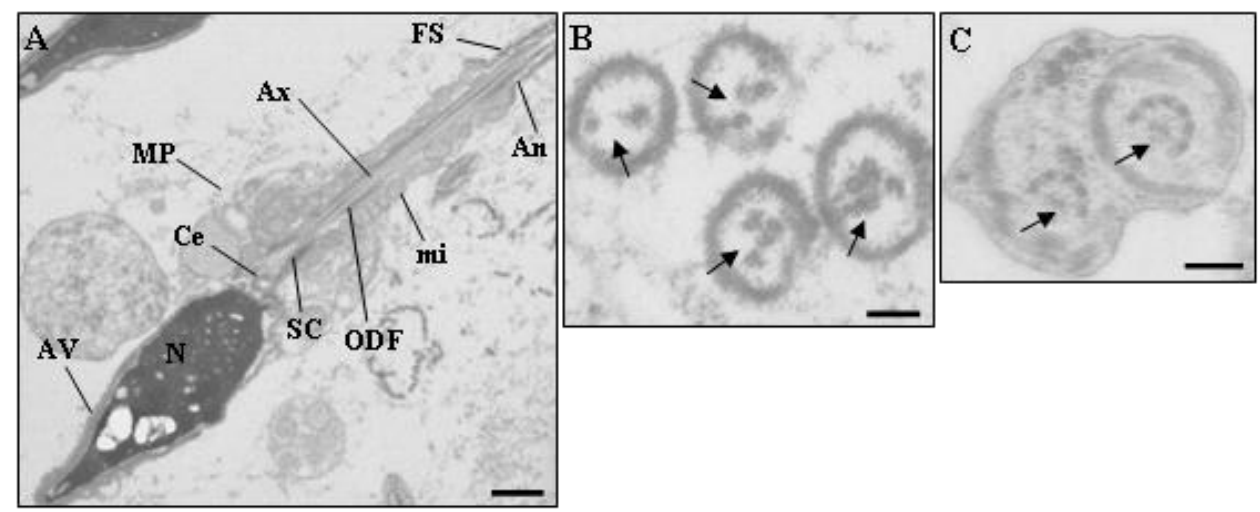

Figure 2. Patient 2 A. Normal spermatozoon. Axoneme (Ax). B,C. Abnormal number and disorganization of the axonemal microtubules (arrows). Bars: $1 \mu \mathrm{m}(\mathrm{A}) ; 0.1 \mu \mathrm{m}(\mathrm{B}, \mathrm{C})$.
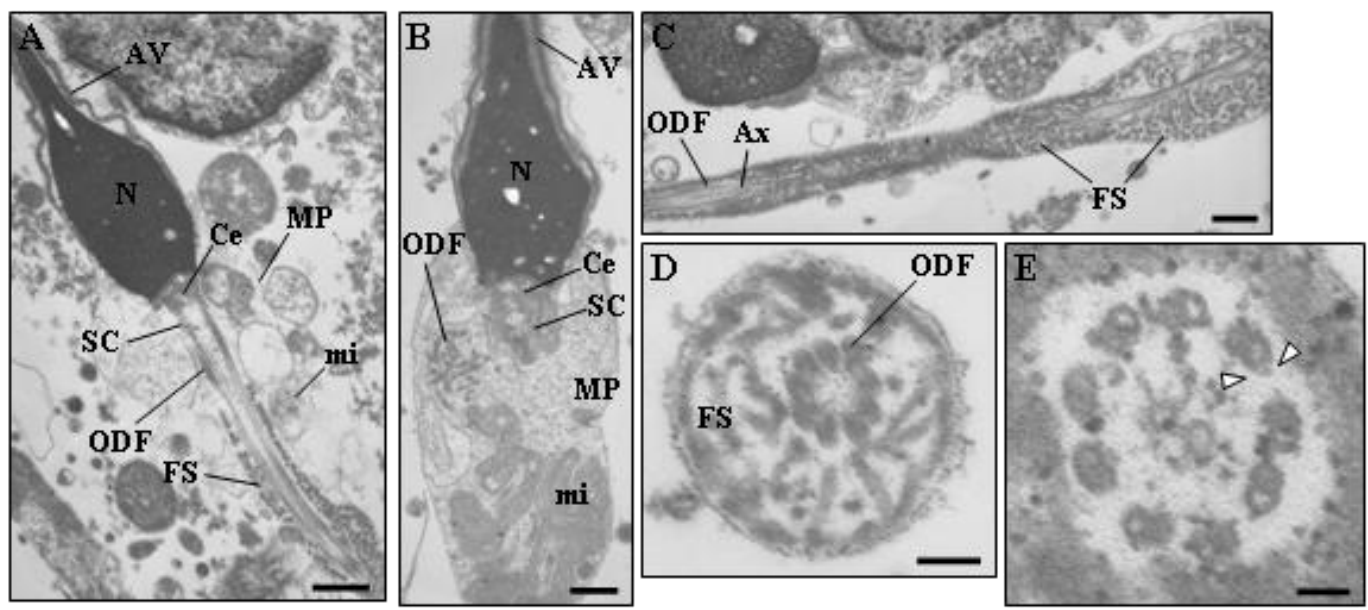

Figure 3. Patient 3 A. Normal nucleus (N), acrosomal vesicle (AV) and centriole region (Ce). Abnormal midpiece (MP). Note swollen mitochondria, absence of the annulus and ascension of the FS. B. Disorganization of the mitochondrial sheath, of the SC and ODF. C, D Disorganization of the FS. E. Absence of dynein arms (arrow-heads). Bars: $1 \mu \mathrm{m}$ (A, B, C); 0.1 $\mu \mathrm{m}$ (D, E). 